\title{
STELLAR MASSES AND STAR FORMATION RATES FOR 1 M GALAXIES FROM SDSS+WISE
}

\author{
Yu-Yen Chang $^{1}$, Arjen van der Wel ${ }^{1}$, Elisabete da Cunha ${ }^{1,2}$, and Hans-Walter Rix ${ }^{1}$ \\ ${ }^{1}$ Max-Planck Institut für Astronomie, Königstuhl 17, D-69117, Heidelberg, Germany; yu-yen.chang@cea.fr \\ ${ }^{2}$ Centre for Astrophysics and Supercomputing, Swinburne University of Technology, Hawthorn, Victoria 3122, Australia \\ Received 2014 November 25; accepted 2015 May 28; published 2015 July 8
}

\begin{abstract}
We combine Sloan Digitital Sky Survey (SDSS) and WISE photometry for the full SDSS spectroscopic galaxy sample, creating spectral energy distributions (SEDs) that cover $\lambda=0.4-22 \mu \mathrm{m}$ for an unprecedentedly large and comprehensive sample of 858,365 present-epoch galaxies. Using MAGPHYS, we then simultaneously and consistently model both the attenuated stellar SED and the dust emission at 12 and $22 \mu \mathrm{m}$, producing robust new calibrations for monochromatic mid-IR star formation rate (SFR) proxies. These modeling results provide the first mid-IR-based view of the bimodality in star formation activity among galaxies, exhibiting the sequence of starforming galaxies ("main sequence") with a slope of $d \log \mathrm{SFR} / d \log M_{*}=0.80$ and a scatter of 0.39 dex. We find that these new SFRs along the SF main sequence are systematically lower by a factor of 1.4 than those derived from optical spectroscopy. We show that for most present-day galaxies, the 0.4-22 $\mu \mathrm{m}$ SED fits can exquisitely predict the fluxes measured by Herschel at much longer wavelengths. Our analysis also illustrates that the majority of stars in the present-day universe are formed in luminous galaxies $\left(\sim L^{*}\right)$ in and around the "green valley" of the color-luminosity plane. We make publicly available the matched photometry catalog and SED modeling results.
\end{abstract}

Key words: catalogs - galaxies: star formation - galaxies: statistics - galaxies: stellar content - infrared: galaxies

Supporting material: machine-readable tables

\section{INTRODUCTION}

Some of the most basic and important constraints on galaxy formation models are the present-day stellar mass function and the distribution of star formation among galaxies with different masses. The Sloan Digitital Sky Survey (SDSS; Blanton et al. 2005; Adelman-McCarthy et al. 2008; Padmanabhan et al. 2008) has provided us with the measurements that underlie our current knowledge of the stellar masses $\left(M_{*}\right)$ and star formation rates (SFRs) of large samples of galaxies (e.g., Brinchmann et al. 2004). SDSS multi-wavelength (ugriz) imaging has been used to estimate luminosities and mass-tolight ratios (e.g., Baldry et al. 2012). The resulting stellar masses have been demonstrated to correlate tightly with total dynamical mass estimates (Taylor et al. 2011) with a scatter of only 0.13 dex. Brinchmann et al. (2004) use the Charlot \& Longhetti (2001) photoionization model to convert nebular emission-line fluxes from SDSS spectroscopy into SFRs. Salim et al. (2007) provided SFRs based on GALEX UV fluxes. However, these previous SDSS studies did not account for the extra information enclosed in dust emission when estimating both SFR and dust attenuation. Here, we present alternative $M_{*}$ and SFR estimates by extending the photometric wavelength coverage of the SDSS spectroscopic sample to the near- and mid-infrared as enabled by WISE (Wright et al. 2010), which provides all-sky coverage at 3-22 $\mu \mathrm{m}$.

The 3.4 and $4.5 \mu \mathrm{m}$ bands sample the Rayleigh-Jeans tail of the stellar spectral energy distribution (SED), which avoids the contribution from hot, young stars which can dominate at shorter wavelenghts. In addition, extinction is usually negligible in these bands. As a result, near-infrared luminosities provide fairly accurate and precise stellar mass estimates, even in the absence of any other photometric or spectroscopic information (Meidt et al. 2014). Many authors have used 3-5 $\mu \mathrm{m}$ photometry from Spitzer or WISE to compare with stellar mass estimates derived from photometry at $2 \mu \mathrm{m}$ and below (e.g., Li et al. 2007; Zhu et al. 2010; Jarrett et al. 2013). Here, we combine SDSS and WISE data to generalize the use of 3-5 $\mu \mathrm{m}$ photometric information for estimating stellar masses.

Mid-infrared emission traces star formation activity through the well-known correlation with polycyclic aromatic hydrocarbon (PAH) emission, sampled by the WISE $12 \mu \mathrm{m}$ band, and through the correlation with thermal radiation from dust, sampled by the WISE $22 \mu \mathrm{m}$ band. A number of authors have compared SFRs from Brinchmann et al. (2004), based on optical emission lines, with mid-IR luminosities from WISE (e.g., Donoso et al. 2012; Lee et al. 2013; Wen et al. 2013, 2014); these studies do not model the mid-IR luminosity to obtain SFRs that are independent of the emission-linebased SFRs.

Here, we take the important step of including the full WISE photometry and employ SED modeling that consistently treats stellar emission along with dust extinction and emission. This will result in more robust masses and star formation rates for dusty galaxies and, in general, an alternative to the emissionline based SFRs from Brinchmann et al. (2004) for the full SDSS spectroscopic galaxy sample. This has not been attempted before for large samples of galaxies. Jarrett et al. (2013) conducted a detailed multi-wavelength study of a small number of objects and provide an important benchmark to test our results. Brown et al. (2014) performed SED fits across the UV to mid-IR wavelength in an exercise similar in approach to what we present here, but for those authors the focus was on producing a set of representative templates: stellar mass and SFR estimates are not presented or discussed. Cluver et al. (2014) analyze the mid-IR properties of the large GAMA sample, but there $\mathrm{H} \alpha$-based SFRs were used as a calibrator for the mid-IR luminosities from WISE. As such, our study is the first to use the mid-IR luminosities of a large sample of galaxies (the full SDSS spectroscopic galaxy sample) to estimate their SFRs in a manner that is entirely independent of optical emission-line luminosities and other external calibrators. 


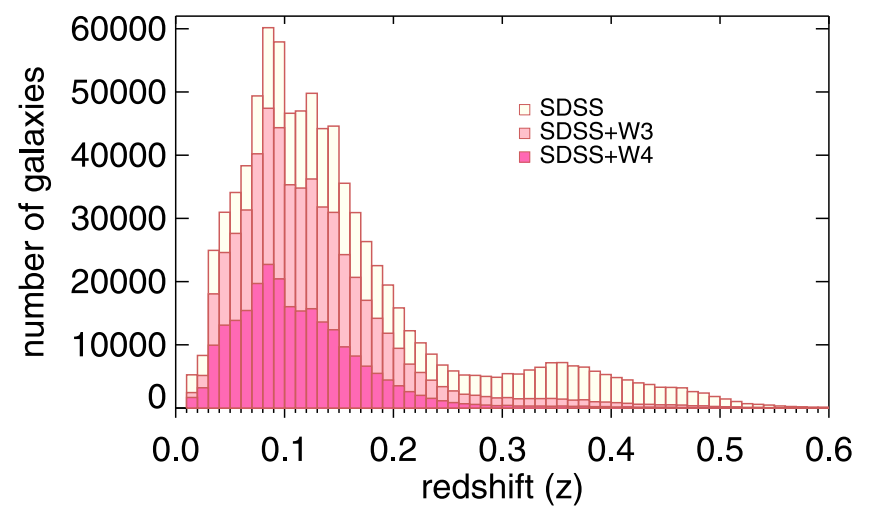

Figure 1. Redshift distribution of the SDSS galaxy sample. $98.24 \%$ of the objects are detected in at least one WISE band. The subsamples with $>2 \sigma$ detections in $W 3(12 \mu \mathrm{m})$ and $W 4(22 \mu \mathrm{m})$ are highlighted.

This paper is organized as follows. In Section 2, we will describe the SDSS and WISE photometric data sets, with a particular focus on total flux measurements for extended sources. In Section 3, we will describe MAGPHYS (da Cunha et al. 2008), the SED fitting code, and test the robustness of the fitting results. In particular, we will test how well MAGPHYS can predict the total IR luminosity using the available wavelength coverage of $0.4-22 \mu \mathrm{m}$. Furthermore, we will compare our $M_{*}$ and SFR estimates with the Brinchmann et al. (2004) measurements. In Section 4, we will describe the publicly released catalog with the fitting results for the entire SDSS spectroscopic sample ( 800,000 galaxies). In Section 5, we will show, as an illustration, how star formation is distributed over galaxies with different masses and colors.

We use AB magnitudes, adopt the cosmological parameters $\left(\Omega_{M}, \Omega_{\Lambda}, h\right)=(0.30,0.70,0.70)$, and adopt the Chabrier (2003) stellar initial mass function.

\section{DATA}

We use the SDSS spectroscopic galaxy sample as compiled in the New York University Value-added Galaxy Catalog (NYU-VAGC; Blanton et al. 2005; Adelman-McCarthy et al. 2008; Padmanabhan et al. 2008), ${ }^{3}$ which contains 858,365 galaxies with reliable redshift measurements (OBJTYPE = GALAXY, Zwarning $=0)$, distributed as shown in Figure 1. We adopt MODELFLUX and MODELFLUX IVAR as the flux measurements and their uncertainties, with galactic extinction corrections as prescribed by Schlafly \& Finkbeiner (2011). To account for the uncertainty in the extinction law, we propagate an uncertainty in $R_{V}=3.1 \pm 0.2$ in our flux uncertainty estimates. Minimum uncertainties are added in quadrature: $0.05 \mathrm{mag}$ for the $\mathrm{u}$ band and $0.02 \mathrm{mag}$ for the griz bands. This is intended to prevent small systematic uncertainties from dominating our goodness-of-fit assessment and the uncertainties in our derived parameters. In case of catastrophic failures in the photometric measurements, which occasionally occur in the $u$ and $z$ bands, we omit these data points from the fits.

The SDSS parent sample is cross-matched with the AllWISE source catalog. ${ }^{4}$ The vast majority of galaxies have counterparts $(98.24 \%)$, identified as the brightest object within a

\footnotetext{
http://sdss.physics.nyu.edu/vagc/

4 http://wise2.ipac.caltech.edu/docs/release/allwise/
}

search radius of 6 arcsec, similar to the WISE PSF. In line with the approach taken by Donoso et al. (2012) and Yan et al. (2013), most matches $(99.08 \%)$ are found within a radius of 3 arcsec and the majority $(91.20 \%)$ do not have multiple matches within 6 arcsec. We provide a flag (FLAG_W) that indicates if there are one or more matches, but we do not exclude any matched sources a priori. Various flux measurements are provided by the AllWISE catalog. In order to minimize the effects of source blending we use the $W$ ? mpro and W? sigmpro flux measurements and their uncertainties. These are profile-fitted photometry measurements, performed simultaneously on neighboring sources, using the point-spread function (PSF) as the source model. Most galaxies with counterparts have significant $W 1$ and $W 2$ flux measurements $(98.83 \%)$. For $W 3$ and $W 4$, many of the flux measurements amount to upper limits $(29.10 \%$ and $70.89 \%$, respectively). The redshift distributions of galaxies with $>2 \sigma \quad W 3$ and $W 4$ detections are shown in Figure 1. We will provide flags to indicate which galaxies have detections and which have upper limits. The usefulness of the upper limits will depend on the goals of the user.

Galaxies are typically smaller than the WISE PSF, but the amount by which W? mpro underestimates the total flux due to the spatial extent of the sources is not necessarily negligible. The $W 1, W 2$, and $W 3$ bands all have very similar PSFs with FWHM $\sim 6-7$ arcsec, while the W4 PSF is larger $(\sim 12)$ arcsec. We investigate the missing light fraction for $W 1, W 2$, and $W 3$ by using galfit to generate (Peng et al. 2010) a series of simulated light distributions with two-dimensional Sérsic profiles convolved with the $W 1$ PSF models provided by Aniano et al. (2011), and inserted into empty sections of real $W 1$ images. ${ }^{5}$ Then, we use galfit to fit the PSF model to the simulated images to measure the PSF profile flux. We find that the difference between the PSF profile flux and the true, total flux is mostly a function of the effective radius of the Sérsic profile, and hardly depends on input flux, axis ratio, or Sérsic index. For a typical size of $5 \operatorname{arcsec}$, we find that $\Delta m$ varies from $15.63 \mathrm{mag}$ for Sérsic index $n=1-15.65 \mathrm{mag}$ for Sérsic index $n=4$. Based on these simulations, we find that the PSF profile magnitude underestimates the total flux by

$$
\begin{aligned}
\Delta m= & 0.10+0.46 \log \left(R_{\mathrm{e}}\right) \\
& +0.47 \log \left(R_{\mathrm{e}}\right)^{2}+0.08 \log \left(R_{\mathrm{e}}\right)^{3},
\end{aligned}
$$

where $R_{\mathrm{e}}$ is the effective radius in arcseconds. This correction is used whenever the size is larger than $R_{\mathrm{e}}=0.5 \operatorname{arcsec}$. We propagate the uncertainty in the adopted radius into Delta $m$, which is in turn propagated into the flux uncertainty.

We use the r-band effective radii and apply a systematic correction of a factor of $1.5 \pm 0.2$ downward to convert from the optical size to near-IR size. This conversion is based on the analysis of Vulcani et al. (2014), who consistently measured sizes for a large set of galaxies across the wavelength range 0.4-2.2 $\mu \mathrm{m}$. These corrected sizes along with Equation (1) are then used to correct the $W 1, W 2$, and $W 3$ fluxes. We use r-band effective radii measured by Simard et al. (2011) when available, and otherwise deVRad or expRad from the SDSS catalog as appropriate. We prefer the Simard et al. (2011) measurements as their fitting methodology (two-dimensional

\footnotetext{
5 http://www.astro.princeton.edu/ ganiano/Kernels/Ker_2012_May/ PSF_fits_Files/
} 
light profiles) is more similar to the methodology used by Vulcani et al. (2014; the SDSS pipeline fits one-dimensional light profiles). We note that the difference between the two versions of our $W 1 / 2 / 3$ magnitude corrections is small: for objects where both measurements are available, the median difference is $0.03 \mathrm{mag}$ and the random scatter $0.14 \mathrm{mag}$. These uncertainties are small compared to the uncertainties in stellar masses and SFRs derived below. These final flux measurements, along with the applied corrections, are listed in the public catalog (see Section 4). The median correction is 0.25 mag with scatters of $-0.07(16 \%$-ile) and $+0.18(84 \%$ ile). Corrections are not made for $W 4$, for which the PSF is twice as wide.

To account for possible systematic uncertainties of the WISE and SDSS photometric systems and further systematic differences between the total flux measurements, we adopt and propagate $0.1 \mathrm{mag}$ uncertainties for all WISE fluxes.

\section{SED MODELING}

\subsection{Method}

We use MAGPHYS to fit the photometric SED (da Cunha et al. 2008, 2012). ${ }^{6}$ The public version of MAGPHYS contains 50,000 stellar population template spectra (the optical photometric library) and 50,000 $\mathrm{PAH}+$ dust emission template spectra (the infrared photometric library). The stellar emission templates use Bruzual \& Charlot (2003) stellar population synthesis models and are generated for a wide range of star formation histories parameterized as exponentially declining models with superimposed random bursts of star formation.

The SEDs are computed by adding the individual spectra of all simple stellar populations weighted by mass. The original MAGPHYS library was constructed for modeling IR-luminous, star-bursting galaxies. The original MAGPHYS library from the public version with 50,000 stellar population template spectra was constructed for modeling star-forming galaxies and we extended the optical library to include 25,000 additional templates for more passive stellar populations.

The infrared templates describe emission by dust. The total dust luminosity over 3-1000 $\mu \mathrm{m}$ has components of emission from PAHs and dust with a range of temperatures. The model contains the ambient (diffuse) interstellar medium and starforming regions (birth clouds). Because stars are born in dense molecular clouds which typically dissipate after $10^{7}$ years, the SEDs of young stellar populations are attenuated both by dust in the birth clouds and dust in the ambient ISM, while the SED of older populations is only attenuated by dust in the diffuse ISM. The absorbed light is assumed to be re-emitted in the midand far-infrared, requiring conservation of energy.

MAGPHYS fits SEDs in the observed frame. Therefore, we generate libraries with model fluxes in the observed frame for a series of narrow redshift bins $(\delta z=0.0001)$ that span the range of our sample. The fitting process for each individual galaxy is then expedited in two ways. First, we only consider optical templates with $(g-i)_{\text {model }}$ colors similar to the observed $(g-i)_{\text {data }}$ color. To be precise, we select templates with $\left|(g-i)_{\text {model }}-(g-i)_{\text {data }}\right|<0.05+\sigma\left[(g-i)_{\text {data }}\right]$, where $\sigma$ refers to the uncertainty. This method works for any two filters, and here we choose $g-i$. The selection is inclusive enough to avoid changes in the results: none of the models eliminated

\footnotetext{
6 http://www.iap.fr/magphys
}

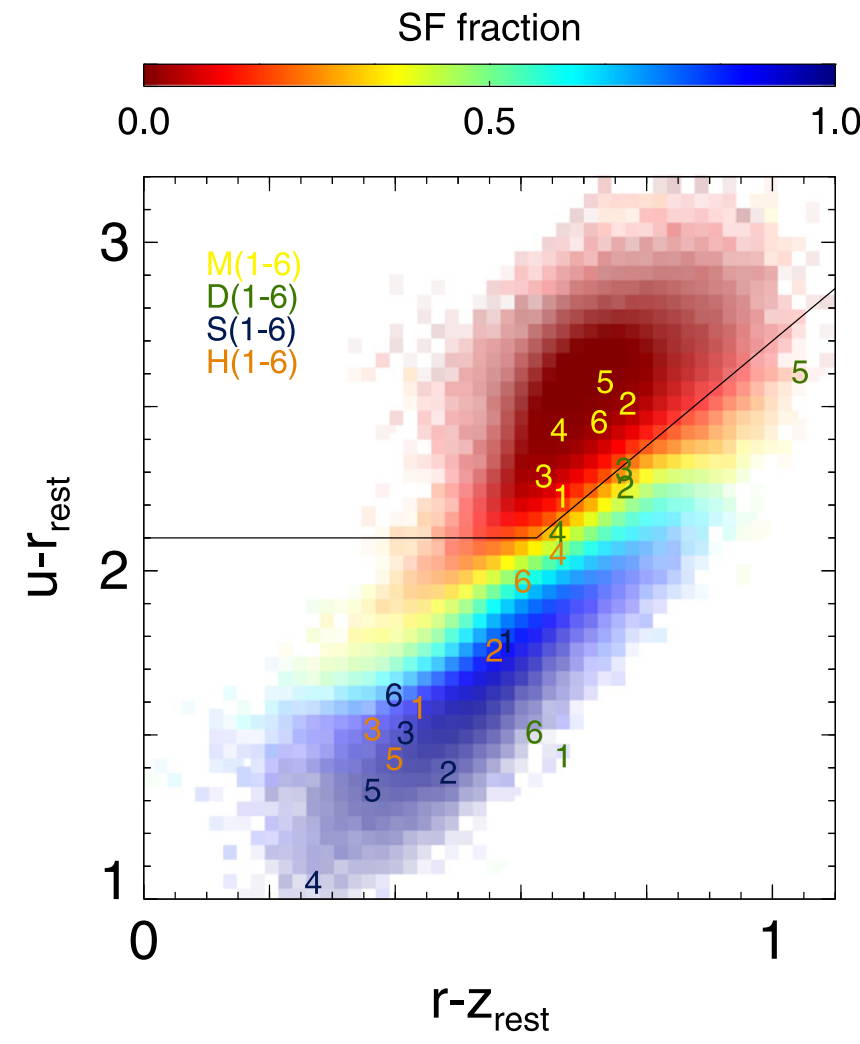

Figure 2. Rest-frame $u-r$ vs. $r-z$ distribution for our SDSS+WISE sample, color-coded according to the fraction of objects that lie on the star-forming sequence or significantly below according to their full SED fit (see Section 4). The clearly distinct color-color distributions of star-forming and quiescent galaxies validate the assumption that a color-color diagram can be effectively used to distinguish the two types of galaxies, as is often done at high redshift. The labels correspond to those objects for which the SEDs are shown in Figures $3-5$ and 7.

from consideration have significant likelihood values. We check that we would get similar results if we fitted the whole model library. Second, we draw a random set of 1000 infrared templates from the total set of 50,000 templates. Since our data do not sample the thermal peak, we cannot stringently constrain the dust temperature distribution, rendering full exploration of the parameter space useless. As a test, we compare the results based on the full and reduced infrared libraries and find no systematic differences (smaller than 0.01 dex for both stellar mass and SFR) in the fitting results and no increases or decreases in the formal fitting uncertainties.

In a small number of cases, we find that MAGPHYS allows the presence of an unrealistically large amount of cold dust. In order to avoid this, we place a mild constraint on the model $250 \mu \mathrm{m}$ luminosity based on the maximum observed $250 \mu \mathrm{m}$ to-22 $\mu \mathrm{m}$ flux ratios (Chary \& Elbaz 2001): $L \nu_{250 \mu \mathrm{m}}\left[L_{\odot} / \mathrm{Hz}\right]$ $<85.7 \times\left(L \nu_{22 \mu \mathrm{m}}+\sigma\left(L \nu_{22 \mu \mathrm{m}}\right)\right)\left[L_{\odot} / \mathrm{Hz}\right]$, which is $\sim 6$ times the typical ratio. We will investigate the precision of our predicted total IR luminosities in Section 3.3.

Given our 10 flux constraints (SDSSugriz; W1-4; $L \nu_{250 \mu \mathrm{m}}$ limit), $\chi^{2}$ is calculated for each model in the library. For each model parameter, the posterior probability distribution can be generated by taking the prior probability distribution (from the full library) and assigning weights $\exp \left(-\chi^{2} / 2\right)$ to each of the models. In doing so, we marginalize over all other model parameters and assume that the likelihood of a given model 

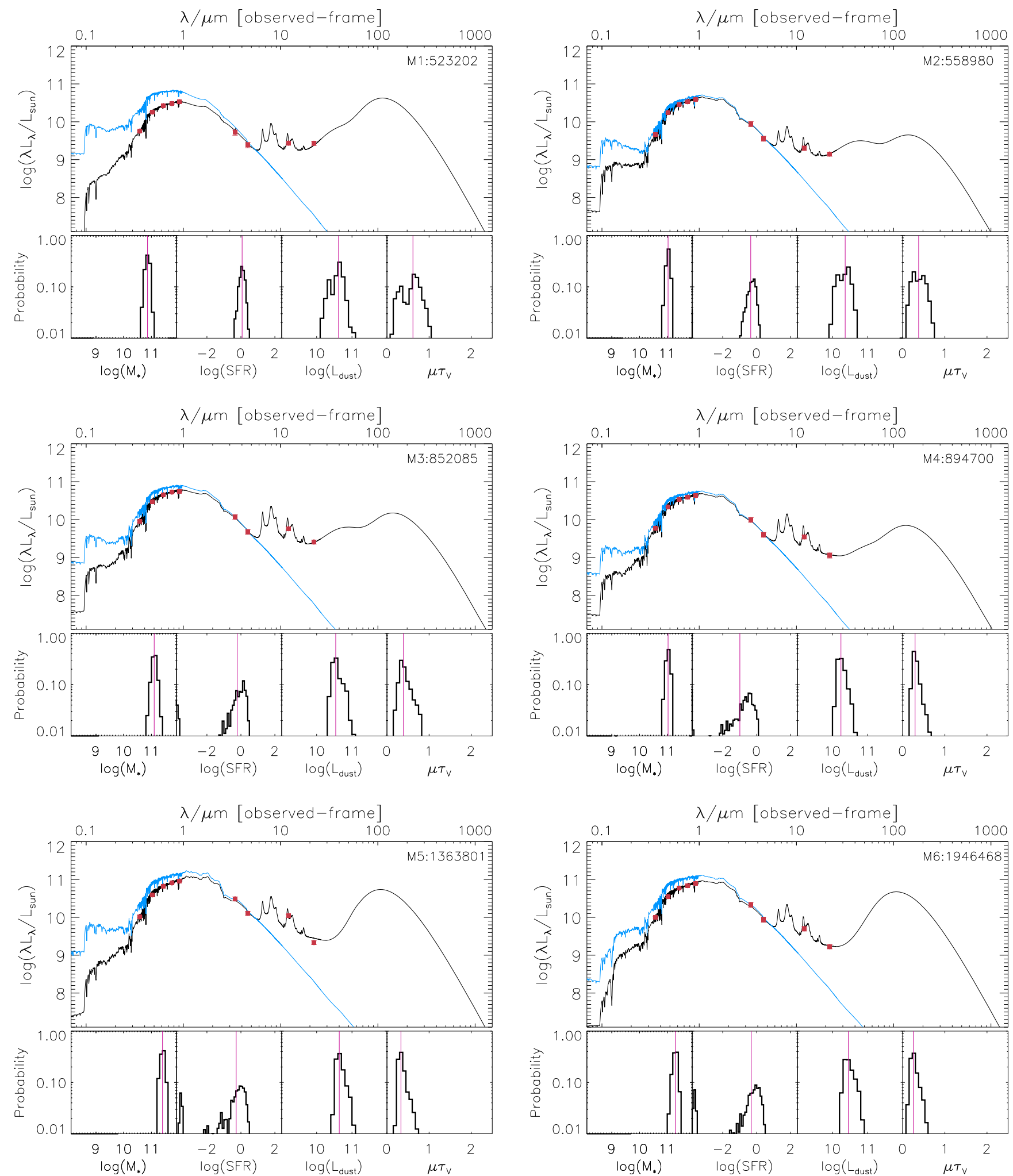

Figure 3. Example SEDs and fitting results of six massive, quiescent galaxies. The large sub-panels show the observed fluxes (red points), the best-fit SEDs (black lines), and the corresponding unattenuated stellar SEDs (blue lines). The smaller panels show the marginalized posterior probability distributions of four models' parameters: $M_{*}$, SFR, dust luminosity (in solar units), and dust attenuation. The red lines show the median values, which we adopt as the best-fitting values of the PDF. The short-hand IDs (M1-6) correspond to the labels in Figure 2.

given the data is proportional to $\exp \left(-\chi^{2} / 2\right)$. We adopt the 50th percentile value-that is, the median-as the best estimate. In practice, in the case of upper limits, we assumed zero flux using the upper limit as the error bar.
Rest-frame fluxes are calculated using the observed fluxes and colors. Analogous to Holden et al. (2012), for each redshift $z$ bin, we derive a linear fit between, on the one hand, observed-frame magnitudes $\left(m_{\mathrm{obs} 1}, m_{\mathrm{obs} 2}\right)$ of the templates in the 

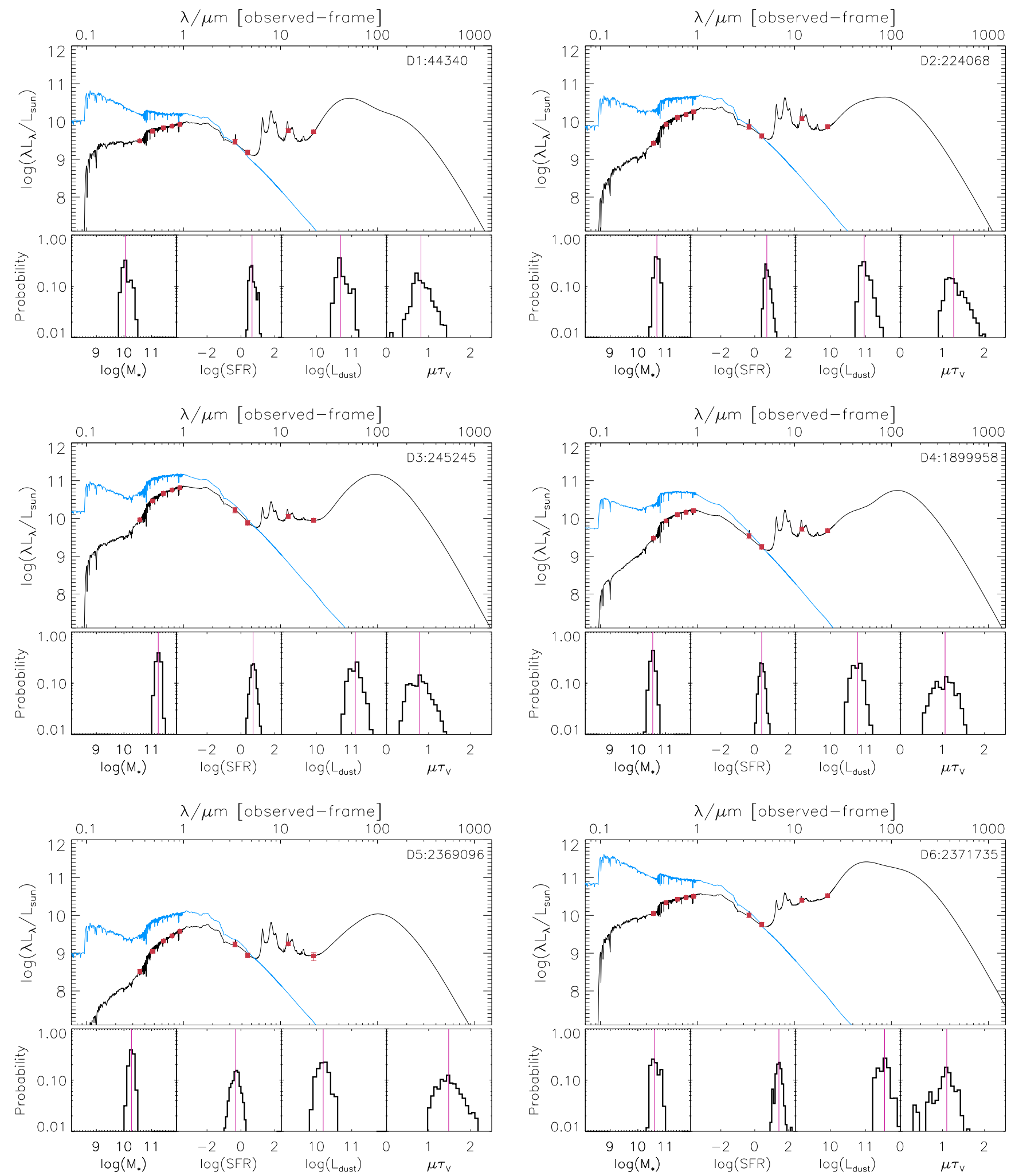

Figure 4. Example SEDs and fitting results of six star-forming galaxies where the optical light is significantly dust-extincted. See Figure 3 for an explanation of the panels, lines, and symbols. The short-hand IDs (D1-6) correspond to the labels in Figure 2.

filters straddling the desired rest-frame band and, on the other hand, the rest-frame magnitude $\left(m_{0}\right)$ of the same templates: $m_{0}=m_{\mathrm{obs} 1}+A(z) \times\left(m_{\mathrm{obs} 1}-m_{\mathrm{obs} 2}\right)+B(z)$. Then, for each galaxy in the sample, we use the observed magnitudes and the values of $A(z)$ and $B(z)$ to compute its rest-frame magnitudes.
We also adopt this technique to calculate the rest-frame 12 and $22 \mu \mathrm{m}$ luminosities. The basic result of the SED MAGPHYS modeling for each object is a joint PDF for the 16 parameters, which we then characterize by the median and the percentiles of the marginalized distributions. 

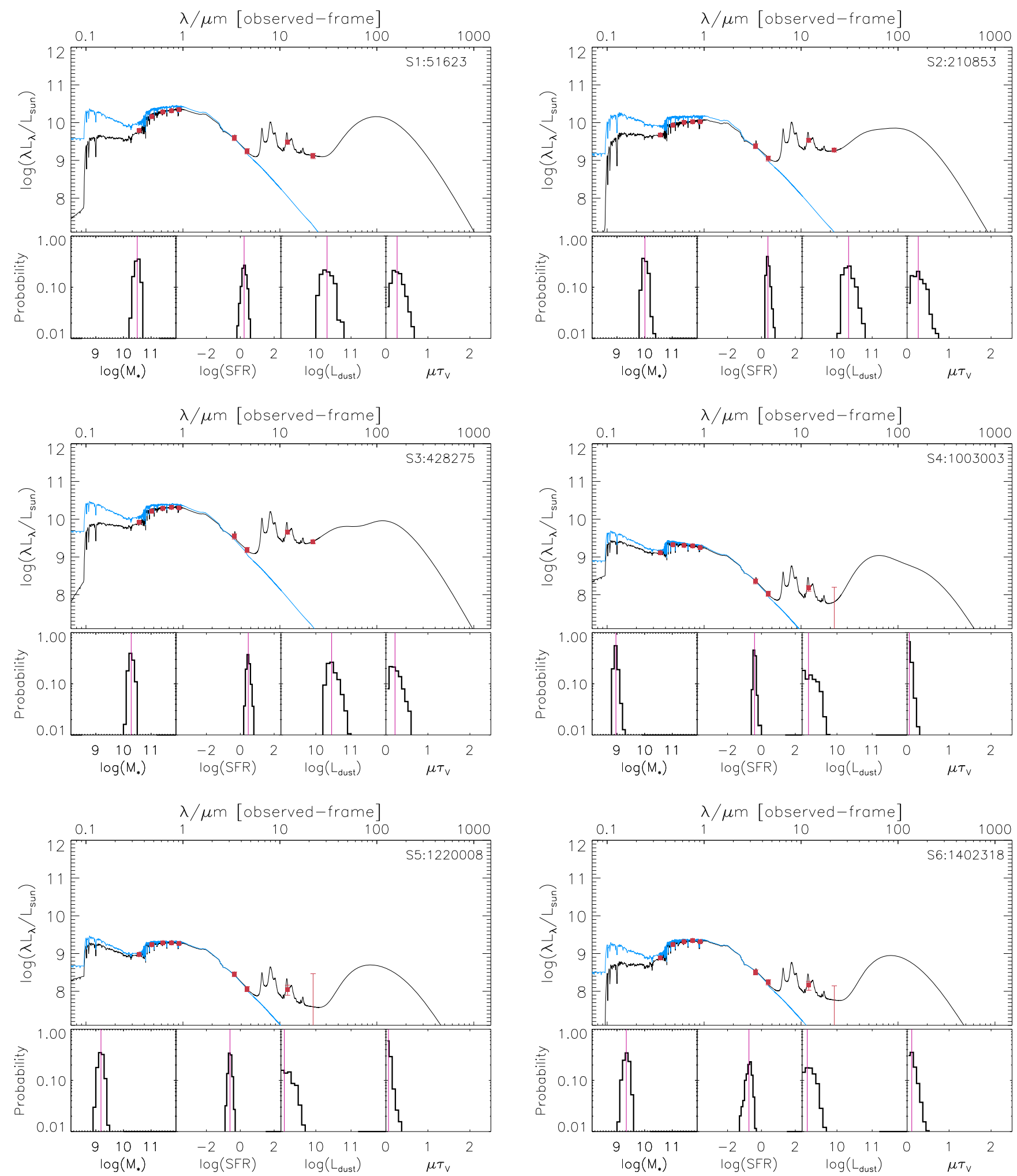

Figure 5. Example SEDs and fitting results of six blue, star-forming galaxies with only modest dust extinction in the optical. See Figure 3 for an explanation of the panels, lines, and symbols. The short-hand IDs (S1-6) correspond to the labels in Figure 2.

\subsection{Sample SEDs}

The galaxies in our sample span a large range in stellar mass, SFR, and colors (Figure 2). In Figures 3-5, we show examples of SEDs and fitting results across the entire parameter space populated by our sample. Figure 3 shows six massive galaxies
$\left(M_{*} \sim 10^{11} M_{\odot}\right)$ with little or no ongoing star formation $\left(\lesssim 1 M_{\odot} \mathrm{yr}^{-1}\right)$. The characteristic red optical SEDs are accompanied by non-neglible mid-IR emission arising from a combination of dust heated by evolved stars and traces of star formation. Figure 4 shows reddened galaxies with significant 
0.0

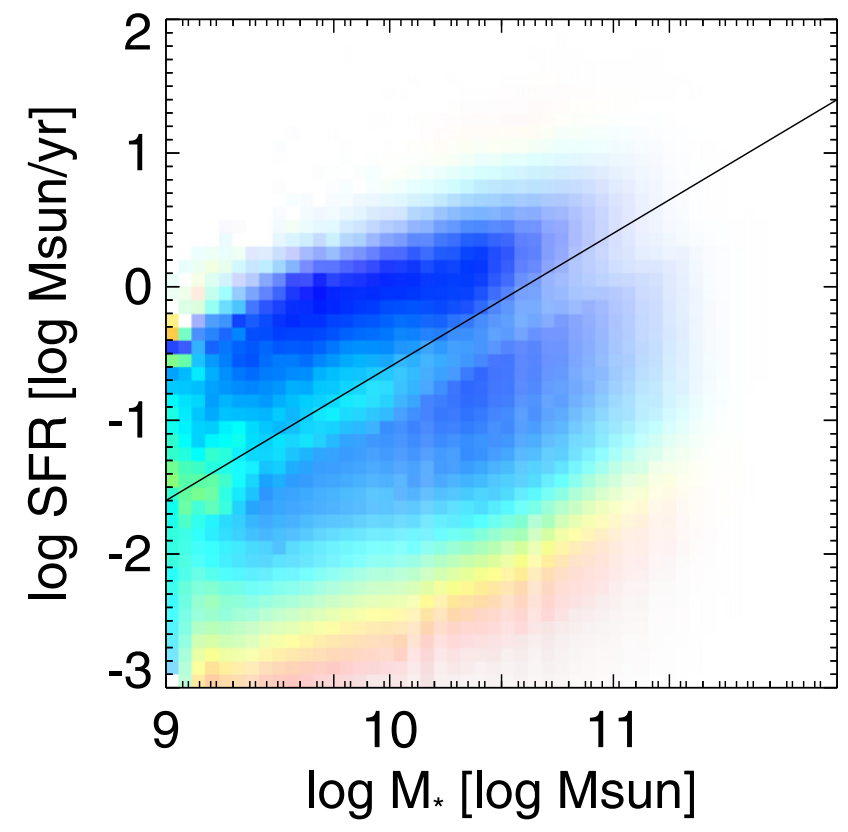

0.3

\section{6}

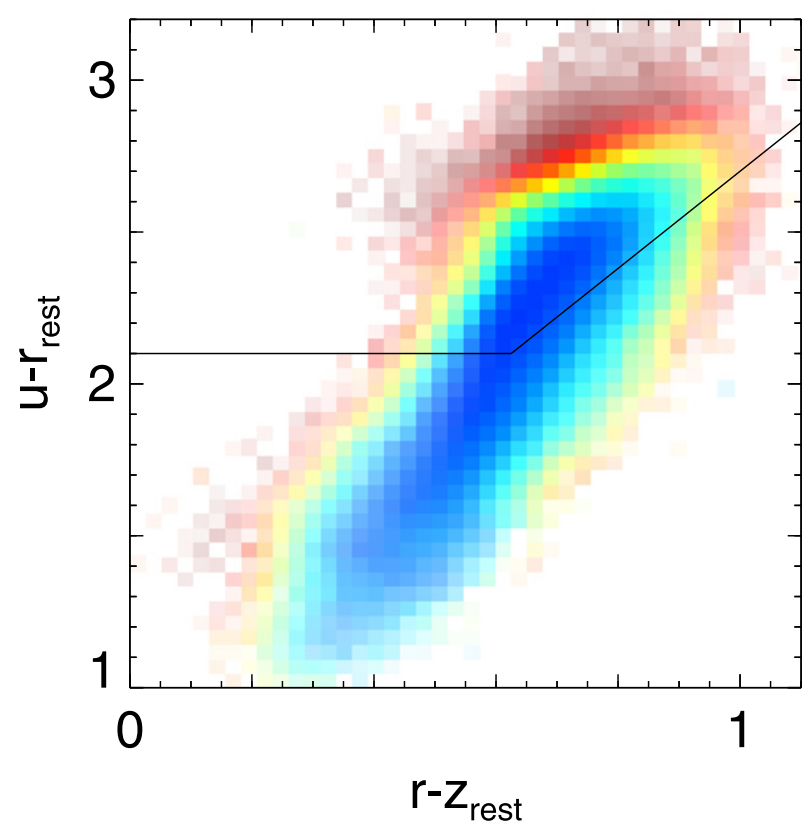

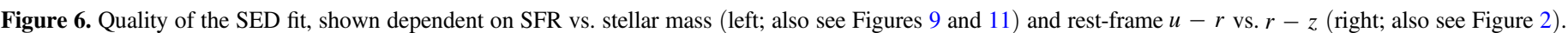

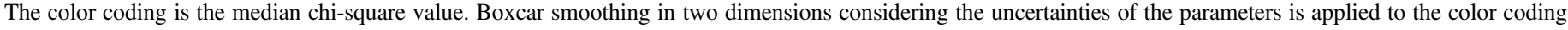
and opacity. For star-forming galaxies, the distribution is about uniform.

star formation activity where the IR luminosity often exceeds the optical/NIR luminosity. Figure 5 shows blue star-forming galaxies with little or no extinction.

To demonstrate the quality of the fits, in Figure 6, we show the chi-square distribution across parameter space $\left(M_{*}, \mathrm{SFR}\right.$, colors). $\chi^{2}$ is the the goodness-of fit parameter for the best-fit model, but not, formally speaking, the reduced chi-squared of the best-fitting model as discussed by Smith et al. (2012). The generally low values of $\chi^{2}$ imply that our library of models is sufficiently extensive.

\subsection{Verification of Total IR Luminosities with Herschel}

MAGPHYS balances the energy absorbed in the UV/optical and the energy released in the IR, such that the inferred total IR luminosity can be expected to be correct if the optical/NIR SED is accurate. Still, since the WISE 12 and $22 \mu \mathrm{m}$ bands do not sample the thermal peak of the dust emission, it is important to test the robustness of the fitting results, in particular, the SFRs and dust luminosities.

Herschel-ATLAS provides PACS and SPIRE photometry over the wavelength range $100-500 \mu \mathrm{m}$. These observations sample the total dust emission more directly than WISE. For the subsample of 285 galaxies that fall within the 16 square degree footprint of the publicly available part of Herschel-ATLAS (Eales et al. 2010; Ibar et al. 2010; Pascale et al. 2011; Rigby et al. 2011; Smith et al. 2011), we verify the precision and accuracy of our inferred total IR luminosity.

Our SDSS+WISE-based models succesfully predict the total IR luminosities and SFRs from SDSS+WISE+Herschel photometry, as illustrated in Figure 7. Even though the marginalized probability distributions for the dust luminosity and SFR are tightened when Herschel photometry is added, we find no systematic offset $(0.00 \mathrm{dex})$ between the SFRs. Furthermore, the scatter $(0.19 \mathrm{dex})$ is consistent with formal confidence intervals (see Figure 8 ). The uncertainties of $\mathrm{SFR} \sim 10^{-4} M_{\odot} \mathrm{yr}^{-1}$ galaxies are large and dust luminosities are poorly constrained.

\subsection{The Public Catalog}

We provide two public catalogs. ${ }^{7}$ They both contain all 858,365 galaxies from the SDSS spectroscopic galaxy sample with good redshift measurements as described in Section 2. Table 1 contains the input data (ID, redshift, fluxes, and Galactic extinction). Table 2 contains the modeling results from MAGPHYS, $V_{\max }$ (see below), rest-frame luminosities, and a set of flags. We recommend using the MAGPHYS modeling results for objects with FLAG $=1(633,205$ out of 858,365). These are all $z<0.2$ galaxies with reliable aperture corrections based on size measurements from Simard et al. (2011; FLAG_R = 1), good WISE photometry (FLAG_W? = 1 or 2 ), and good-quality SED fits (FLAG_CHI2 = 1). This primary sample contains $91.40 \%$ of all SDSS galaxies at $z<0.2$.

Our $V_{\max }$ calculation only includes the maximum redshift at which galaxies would be retained in the sample. No lowredshift limit, related to the SDSS bright-magnitude limit, is taken into account. For the purpose of illustrating the distribution of SFR and stellar mass, this does not matter given the large volume, but for individual low-redshift, highluminosity galaxies, the user should keep in mind that our $V_{\max }$ cannot be directly used.

\footnotetext{
http://irfu.cea.fr/pisp/yu-yen.chang/sw.html
} 

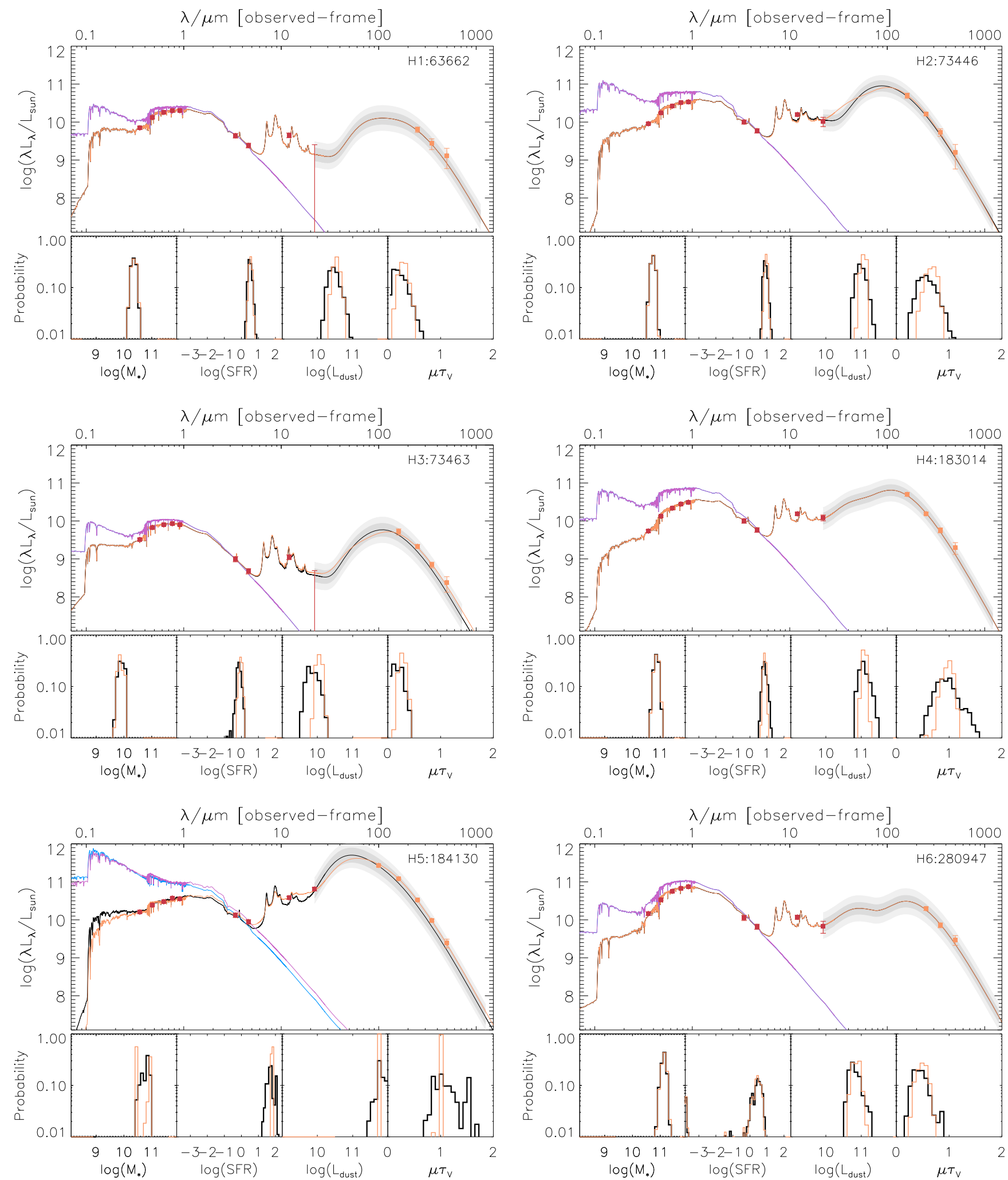

Figure 7. Example SEDs and fitting results of six galaxies with Herschel-ATLAS photometry available (orange points) in addition to the SDSS and WISE (red points). The blue and black lines represent fitting results to the SDSS+WISE data only (as in Figure 3), and the dark (light) gray areas represent the 68\% (95\%) confidence intervals for the far-IR dust SED predicted from these fits. The magenta and orange lines represent the best-fitting unattenuated and attenuated SEDs, respectively, when the Herschel-ATLAS SEDs are also simultaneously fit. The smaller panels show the marginalized posterior probability distributions of four of the model parameters. In all cases, the SDSS+WISE fitting results predict the Herschel photometry well and all of the results inferred without Herschel data are consistent with the results inferred with Herschel data. However, the constraints on the dust luminosity and dust attenuation parameter are tightened once Herschel photometry is included. The short-hand IDs (H1-6) correspond to the labels in Figure 2. 


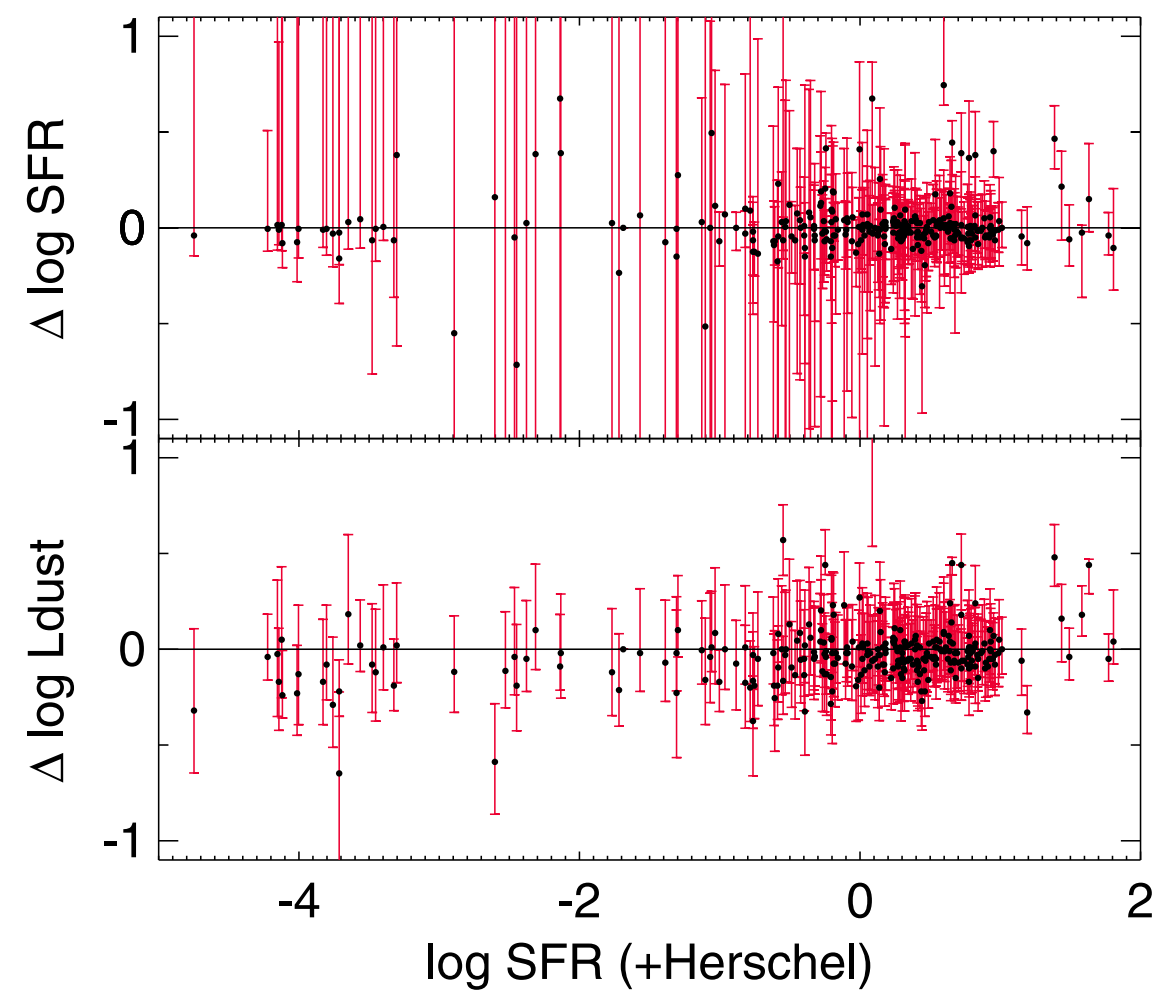

Figure 8. Top: SFR from the SED fits with only optical (SDSS) and near/mid-IR (WISE) data minus SFR from fits that also include far-IR Herschel data vs. SFR from fits with Herschel data. The objects included here are those that overlap between our SDSS-selected sample and Herschel-ATLAS. Red error bars indicate $68 \%$ confidence intervals. Bottom: Same as above, but for dust luminosity. Our SDSS+WISE-based fitting results are generally consistent with the SDSS+WISE+Herschel-based fitting results.

\section{DISCUSSION}

\subsection{Comparison with Brinchmann et al. (2004)}

Stellar masses and SFRs from the MPA-JHU catalogs (Brinchmann et al. 2004) are widely used. The differences between their stellar mass estimates and ours are the addition of WISE 3.4 and $4.5 \mu \mathrm{m}$ photometry as tracers of stellar mass and the updated Galactic extinction correction from Schlafly \& Finkbeiner (2011). Furthermore, the simultaneous fitting of mid-IR photometry provides a fundamentally different constraint on the dust properties of galaxies than optical photometry and emission-line ratios. In addition, dust attenuation laws are also different. In Brinchmann et al. (2004), the adopted slope for the attenuation curve is -0.7 , following Charlot \& Fall (2000). MAGPHYS has the same slope for diffuse dust, but a steeper slope $(-1.3)$ for birth clouds. Despite these differences, offsets between the two sets of mass estimates are small and insignificant (left-hand panel, Figure 9). The reasons for this are that the same stellar population models and star formation histories are used and that the impact of the WISE photometry is limited due to the relatively large uncertainties on the total flux measurements.

Larger differences are seen for the SFR estimates. As opposed to the stellar mass estimates, the SFR estimates rely on wholly different tracers in the two cases. Selecting star-forming galaxies by their location in the color-color diagram (Figure 2), we find a median offset across the sample of 0.22 dex and a scatter of $\sim 0.3$ dex. This offset does not depend on mass or redshift, or even on whether the galaxies have significant 12 and $22 \mu \mathrm{m}$ detections, but strongly varies with SFR (Figure 9); for high-SFR galaxies, our values are large compared to Brinchmann et al. (2004) while for low-SFR galaxies, our values are small. The anti-correlation between the changes in $M_{*}$ and SFR is the result of the underlying anti-correlation between the mass-to-light ratio and sSFR.

\subsection{2 and $22 \mu \mathrm{m}$ Luminosities as SFR Indicators}

Another consequence is that our 12 and $22 \mu \mathrm{m}$ SFR conversions are lower by, respectively, 0.22 and $0.10 \mathrm{dex}$ compared to the conversion based on a comparison with the Brinchmann et al. (2004) estimates, as carried out by Lee et al. (2013).

In Figure 10, we show the correlations between the 12 and $22 \mu \mathrm{m}$ luminosities and our SFR estimates. The large downward scatter is due to mid-IR radiation that is not associated with star formation. In particular, the mid-IR luminosities of quiescent galaxies do not reflect star formation activity, but rather circumstellar dust and PAHs heated by evolved stars (e.g., Bressan et al. 2006). Even for many galaxies that are starforming according to our definition-separated in color-color space (Figure 2; SF galaxies: $(u-r)_{\text {rest }}<2.1$ or $\left.(u-r)_{\text {rest }}<1.6 \times(r-z)_{\text {rest }}+1.1\right)$-a large fraction of the mid-IR luminosity is not attributed to star formation. Keeping this in mind, we derive the following conversions from mid-IR luminosity to SFR:

$$
\log \operatorname{SFR} /\left(M_{\odot} \mathrm{yr}^{-1}\right)=\log L_{12} / L_{\odot}-9.18
$$

and

$$
\log \mathrm{SFR} /\left(M_{\odot} \mathrm{yr}^{-1}\right)=\log L_{22} / L_{\odot}-9.08 .
$$

These relationships are determined by calculating the median values of the SFR in bins of 0.25 dex in luminosity and performing a linear fit to these median values, weighting by the inverse of the square root of the numer of objects in the bins. 
Table 1

Input Catalog

\begin{tabular}{|c|c|c|c|}
\hline Column Name & Format & Unit & Column Description \\
\hline ID & LONG & $\ldots$ & NYU-VAGC catalog index \\
\hline R.A. & DOUBLE & degree & J2000 R.A. [degree] from NYU-VAGC (r band) \\
\hline Decl. & DOUBLE & degree & J2000 Decl. [degree] from NYU-VAGC ( $\mathrm{r}$ band) \\
\hline REDSHIFT & DOUBLE & $\ldots$ & Redshift from the NYU-VAGC spectroscopic catalog \\
\hline PLATE & LONG & $\cdots$ & SDSS plate from NYU-VAGC \\
\hline MJD & LONG & $\cdots$ & SDSS mjd from NYU-VAGC \\
\hline FIBERID & LONG & $\ldots$ & SDSS fiberid from NYU-VAGC \\
\hline DESIGNATION & STRING & $\ldots$ & ALLWISE designation \\
\hline FLUX0_U & DOUBLE & Jy & u-band Flux before corrections \\
\hline FLUX0_U_E & DOUBLE & Jy & Uncertainty of u-band flux before corrections \\
\hline FLUX0_G ${ }^{-}$ & DOUBLE & Jy & $g$-band flux before corrections \\
\hline FLUX0_G_E & DOUBLE & Jy & Uncertainty of $g$-band flux before corrections \\
\hline FLUX0_R & DOUBLE & Jy & r-band flux before corrections \\
\hline FLUX0_R_E & DOUBLE & Jy & Uncertainty of r-band flux before corrections \\
\hline FLUX0_I & DOUBLE & Jy & $i$-band flux before corrections \\
\hline FLUX0_I_E & DOUBLE & Jy & Uncertainty of $i$-band flux before corrections \\
\hline FLUX0_Z- Z & DOUBLE & Jy & $z$-band flux before corrections \\
\hline FLUX0_Z_E & DOUBLE & Jy & Uncertainty of $z$-band flux before corrections \\
\hline FLUX0_W & DOUBLE & Jy & W1-band flux before corrections \\
\hline FLUX0_W1_E & DOUBLE & Jy & Uncertainty $W 1$-band of flux before corrections \\
\hline FLUX0 ${ }^{-} \mathrm{W}^{-}$ & DOUBLE & Jy & W2-band flux before corrections \\
\hline FLUX0_W2_E & DOUBLE & Jy & Uncertainty $W 2$-band of flux before corrections \\
\hline FLUX0_W3 & DOUBLE & Jy & W3-band flux before corrections \\
\hline FLUX0_W3_E & DOUBLE & Jy & Uncertainty $W 3$-band of flux before corrections \\
\hline FLUX0_W4 & DOUBLE & Jy & W4-band flux before corrections \\
\hline FLUX0 ${ }^{-} \mathrm{W} 4 \mathrm{E}$ & DOUBLE & Jy & Uncertainty W4-band of flux before corrections \\
\hline FLUX_U & DOUBLE & Jy & u-band Flux after corrections \\
\hline FLUX_U_E & DOUBLE & Jy & Uncertainty of u-band flux after corrections \\
\hline FLUX_G & DOUBLE & Jy & $g$-band Flux after corrections \\
\hline FLUX_G_E & DOUBLE & Jy & Uncertainty of $g$-band flux after corrections \\
\hline FLUX_R ${ }^{-}$ & DOUBLE & Jy & r-band Flux after corrections \\
\hline FLUX_R_E & DOUBLE & Jy & Uncertainty of r-band flux after corrections \\
\hline FLUX_I & DOUBLE & Jy & $i$-band Flux after corrections \\
\hline FLUX_I_E & DOUBLE & Jy & Uncertainty of $i$-band flux after corrections \\
\hline FLUX_Z & DOUBLE & Jy & $z$-band Flux after corrections \\
\hline FLUX_Z_E_E & DOUBLE & Jy & Uncertainty of $z$-band flux after corrections \\
\hline FLUX_W- ${ }_{-}^{-} 1$ & DOUBLE & Jy & W1-band Flux after corrections \\
\hline FLUX_W1_E & DOUBLE & Jy & Uncertainty $W 1$-band of flux after corrections \\
\hline FLUX_W2 & DOUBLE & Jy & W2-band Flux after corrections \\
\hline FLUX_W2_E & DOUBLE & Jy & Uncertainty $W 2$-band of flux after corrections \\
\hline FLUX_W3 ${ }^{-}$ & DOUBLE & Jy & W3-band Flux after corrections \\
\hline FLUX_W3_E & DOUBLE & Jy & Uncertainty $W 3$-band of flux after corrections \\
\hline FLUX_W4 & DOUBLE & Jy & W4-band Flux after corrections \\
\hline FLUX_W4_E & DOUBLE & Jy & Uncertainty W4-band of flux after corrections \\
\hline EXTIN_ŪU & DOUBLE & mag & Galactic extinction correction for u band \\
\hline EXTIN_G & DOUBLE & mag & Galactic extinction correction for $g$-band \\
\hline EXTIN_R & DOUBLE & mag & Galactic extinction correction for $\mathrm{r}$ band \\
\hline EXTIN_I I & DOUBLE & mag & Galactic extinction correction for $i$-band \\
\hline EXTIN_Z Z & DOUBLE & mag & Galactic extinction correction for $z$-band \\
\hline
\end{tabular}

(This table is available in its entirety in machine-readable form.)

The upward scatter (84\%-ile minus median) in SFR is 0.20 dex, while the downward scatter (median minus 16\%ile) is $0.60 \mathrm{dex}$, reflecting non-SF contributions. The scatter obviously depends on luminosity: above $10^{10} L_{\odot}$, the downward scatter is also 0.30 dex.

The 12 and $22 \mu \mathrm{m}$ values are 0.13 and 0.04 dex higher than the conversions provided by Jarrett et al. (2013), who based their SFRs on GALEX UV fluxes and the standard Spitzerl MIPS $24 \mu \mathrm{m}$ calibration from Rieke et al. (2009). The relatively good agreement with the Jarrett et al. (2013) calibrations is encouraging, as their sample consists of 17 well-resolved, nearby galaxies for which measurement uncertainties are minimal. (In fact, the small difference is consistent with the random variation expected on average for a small 
Table 2

Output Catalog

\begin{tabular}{|c|c|c|c|}
\hline Column Name & Format & Unit & Column Description \\
\hline LMASS_2_5 & FLOAT & $\log M_{\odot}$ & log stellar mass ( 2.5 th percentile) \\
\hline LMASS_16 & FLOAT & $\log M_{\odot}$ & log stellar mass (16th percentile) \\
\hline LMASS_50 & FLOAT & $\log M_{\odot}$ & log stellar mass (50th percentile) \\
\hline LMASS_84 & FLOAT & $\log M_{\odot}$ & log stellar mass (84th percentile) \\
\hline LMASS_97_5 & FLOAT & $\log M_{\odot}$ & log stellar mass (97.5th percentile) \\
\hline LSFR_2_5 & FLOAT & $\log M_{\odot} \mathrm{yr}^{-1}$ & $\log$ SFR (2.5th percentile) \\
\hline LSFR_16 & FLOAT & $\log M_{\odot} \mathrm{yr}^{-1}$ & log SFR (16th percentile) \\
\hline LSFR_50 & FLOAT & $\log M_{\odot} \mathrm{yr}^{-1}$ & $\log$ SFR (50th percentile) \\
\hline LSFR_84 & FLOAT & $\log M_{\odot} \mathrm{yr}^{-1}$ & log SFR (84th percentile) \\
\hline LSFR_97_5 & FLOAT & $\log M_{\odot} \mathrm{yr}^{-1}$ & $\log$ SFR (97.5th percentile) \\
\hline LSSFR_2_5 & FLOAT & $\log 1 \mathrm{yr}^{-1}$ & log specific SFR (2.5th percentile) \\
\hline LSSFR_1 $1 \overline{6}$ & FLOAT & $\log 1 \mathrm{yr}^{-1}$ & log specific SFR (16th percentile) \\
\hline LSSFR_50 & FLOAT & $\log 1 \mathrm{yr}^{-1}$ & log specific SFR (50th percentile) \\
\hline LSSFR_84 & FLOAT & $\log 1 \mathrm{yr}^{-1}$ & log specific SFR (84th percentile) \\
\hline LSSFR_97_5 & FLOAT & $\log 1 \mathrm{yr}^{-1}$ & log specific SFR (97.5th percentile) \\
\hline LDUST_2_5 & FLOAT & $\log L_{\odot}$ & $\log$ dust luminosity (2.5th percentile) \\
\hline LDUST_16 & FLOAT & $\log L_{\odot}$ & log dust luminosity (16th percentile) \\
\hline LDUST_50 & FLOAT & $\log L_{\odot}$ & $\log$ dust luminosity (50th percentile) \\
\hline LDUST_84 & FLOAT & $\log L_{\odot}$ & $\log$ dust luminosity (84th percentile) \\
\hline LDUST_97_5 & FLOAT & $\log L_{\odot}$ & log dust luminosity (97.5th percentile \\
\hline MU_2_5 & FLOAT & $\cdots$ & dust attenuation parameter in da Cunha et al. (2008) (2.5th percentile) \\
\hline MU_16 & FLOAT & $\cdots$ & dust attenuation parameter in da Cunha et al. (2008) (16th percentile) \\
\hline MU_50 & FLOAT & $\cdots$ & dust attenuation parameter in da Cunha et al. (2008) (50th percentile) \\
\hline MU_84 & FLOAT & $\cdots$ & dust attenuation parameter in da Cunha et al. (2008) (84th percentile) \\
\hline MU_97_5 & FLOAT & $\cdots$ & dust attenuation parameter in da Cunha et al. (2008) (97.5th percentile) \\
\hline TAUV_2_5 & FLOAT & $\cdots$ & dust attenuation parameter in da Cunha et al. (2008) (2.5th percentile) \\
\hline TAUV_16 & FLOAT & $\cdots$ & dust attenuation parameter in da Cunha et al. (2008) (16th percentile) \\
\hline TAUV_50 & FLOAT & $\cdots$ & dust attenuation parameter in da Cunha et al. (2008) (50th percentile) \\
\hline TAUV_84 & FLOAT & $\cdots$ & dust attenuation parameter in da Cunha et al. (2008) (84th percentile) \\
\hline TAUV_97_5 & FLOAT & $\cdots$ & dust attenuation parameter in da Cunha et al. (2008) (97.5th percentile) \\
\hline V_MAX & DOUBLE & $\mathrm{Mpc}^{3}$ & Maximum volume for LMASS_50 \\
\hline LREST_U & DOUBLE & $\log L_{\odot}$ & Rest-frame u-band Luminosity \\
\hline LREST_G & DOUBLE & $\log L_{\odot}$ & Rest-frame $g$-band Luminosity \\
\hline LREST_R & DOUBLE & $\log L_{\odot}$ & Rest-frame r-band Luminosity \\
\hline LREST_I & DOUBLE & $\log L_{\odot}$ & Rest-frame $i$-band Luminosity \\
\hline LREST_Z & DOUBLE & $\log L_{\odot}$ & Rest-frame $z$-band Luminosity \\
\hline LREST_W1 & DOUBLE & $\log L_{\odot}$ & Rest-frame $W 1$-band Luminosity \\
\hline LREST_W2 & DOUBLE & $\log L_{\odot}$ & Rest-frame $W 2$-band Luminosity \\
\hline LREST_W3 & DOUBLE & $\log L_{\odot}$ & Rest-frame W3-band Luminosity \\
\hline LREST_W4 & DOUBLE & $\log L_{\odot}$ & Rest-frame W4-band Luminosity \\
\hline FLAG_R & INT & $\cdots$ & 1: Simard radius; 2: deVaucouleurs radius; 3 : exponential radius; 0: no radius \\
\hline FLAG_W & INT & $\cdots$ & 1: single optical matched in WISE; $2:>1$ counterparts within $6^{\prime \prime} ; 0:$ not matched \\
\hline FLAG_W1 & INT & $\cdots$ & 1: detected $W 1(\mathrm{SNR}>2) ; 2$ : upper limit on $W 1 ; 0$ : no $W 1$ data \\
\hline FLAG_W2 & INT & $\cdots$ & 1: detected $W 2($ SNR $>2) ; 2$ : upper limit on $W 2 ; 0$ : no $W 2$ data \\
\hline FLAG_W3 & INT & $\cdots$ & 1: detected $W 3(\mathrm{SNR}>2)$; 2 : upper limit on $W 3$; 0 : no $W 3$ data \\
\hline FLAG_W4 & INT & $\cdots$ & 1: detected $W 4($ SNR $>2) ; 2$ : upper limit on $W 4 ; 0$ : no $W 4$ data \\
\hline FLAG_CHI2 & INT & $\cdots$ & 1: $\chi^{2}<3$ for best-fit model; $0: \chi^{2}>3$ for best-fit model \\
\hline FLAG & INT & $\ldots$ & 1: good fit $($ FLAG_R $=1$; FLAG_W? $=1$ or 2 ; FLAG_CHI2 $=1 ; z<0.2)$; 0: others \\
\hline
\end{tabular}

sample of 17 objects.) Our calibration generalizes their result by extending the dynamic range in stellar mass and SFR by an order of magnitude upward and using a sample of several hundred thousand galaxies.

Our 12 and $22 \mu \mathrm{m}$ SFR conversions are lower by, respectively, 0.22 and $0.11 \mathrm{dex}$ compared to Lee et al.
(2013), who use dust-corrected $H \alpha$ luminosity-based SFRs. ${ }^{8}$ Part of this offset can be attributed to the systematic differences between our SFR estimates and those of Brinchmann et al.

\footnotetext{
8 These differences include the appropriate correction from Salpeter to Chabrier IMF for the Lee et al. (2013) conversions.
} 

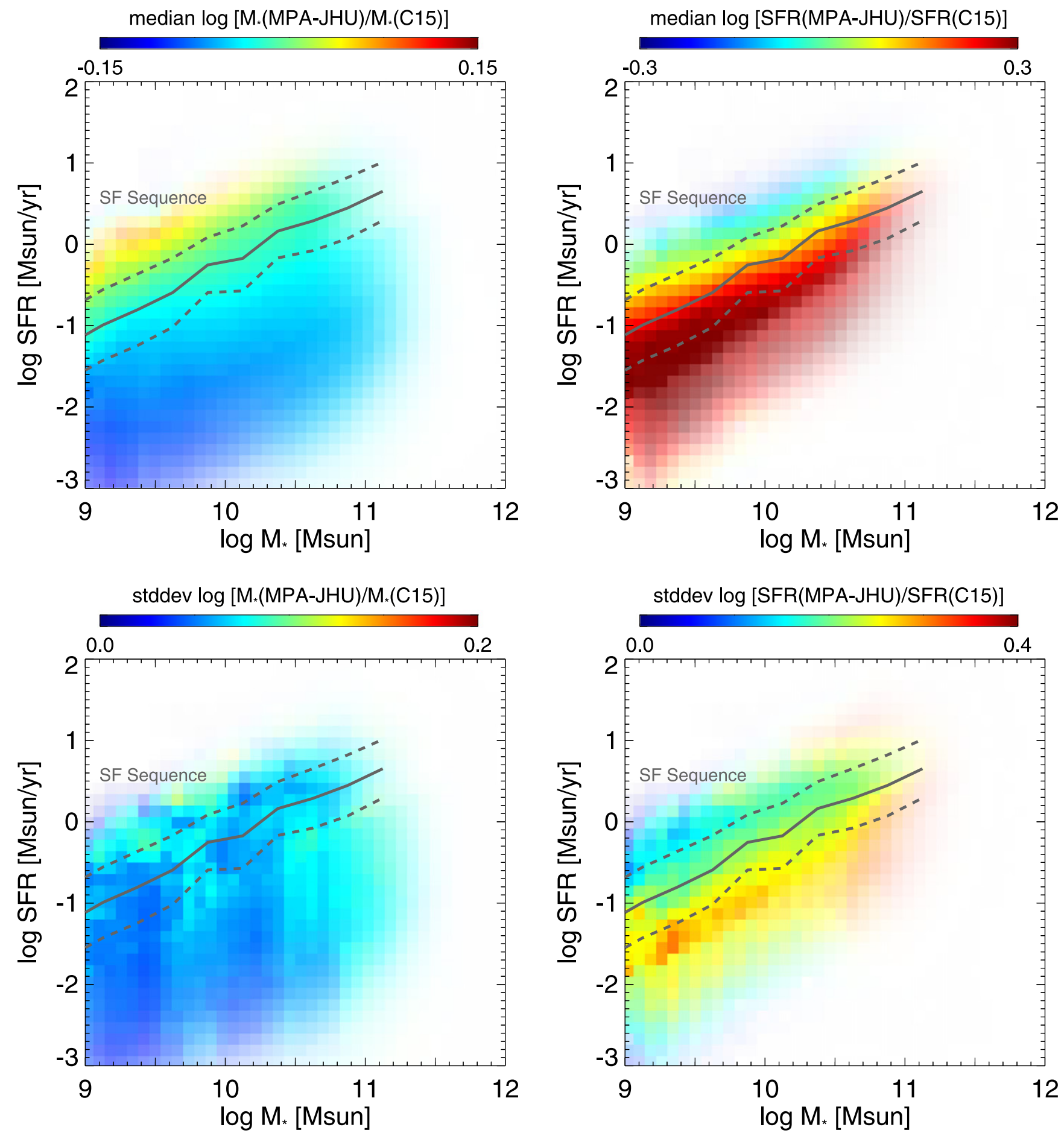

Figure 9. Comparison between the MPA-JHU values for $M_{*}$ and SFR and the values derived here (C15). Top left panel: median difference between the stellar mass estimates. These are always small $(\lesssim 0.10 \mathrm{dex})$. Bottom left panel: scatter in the stellar mass estimates; generally, $\lesssim 0.15$ dex. Top right panel: median difference in SFR. Here, we only show star-forming galaxies selected by the color-color classification given in the right-hand panel of Figure 2. Across the sample the offset is 0.22 dex. It is strongly SFR-dependent and ranges from -0.3 dex to +0.3 dex. Bottom right panel: scatter in the SFR estimates, typically 0.3 dex or a factor of two. The gray lines show the star-forming sequence in Figure 11.

(2004; see Section 4.1). Additional factors are the differences in sample selection and modeling technique. We select starforming galaxies on the basis of their optical colors, whereas Lee et al. (2013) select galaxies with large $22 \mu \mathrm{m}$ luminosities. Moreover, not all mid-IR radiation traces SF, which is taken into account in our modeling, whereas Lee et al. (2013) simply use total mid-IR luminosity as a SF tracer.

\subsection{A Fresh View on Bimodality}

Assessing bimodality in the galaxy population requires a sample that is corrected for completeness and sufficient sensitivity in the star formation tracer to separate star-forming and passive galaxies. We compare the magnitude in the $r$ band with the WISE bands at different redshift and find that the mass 

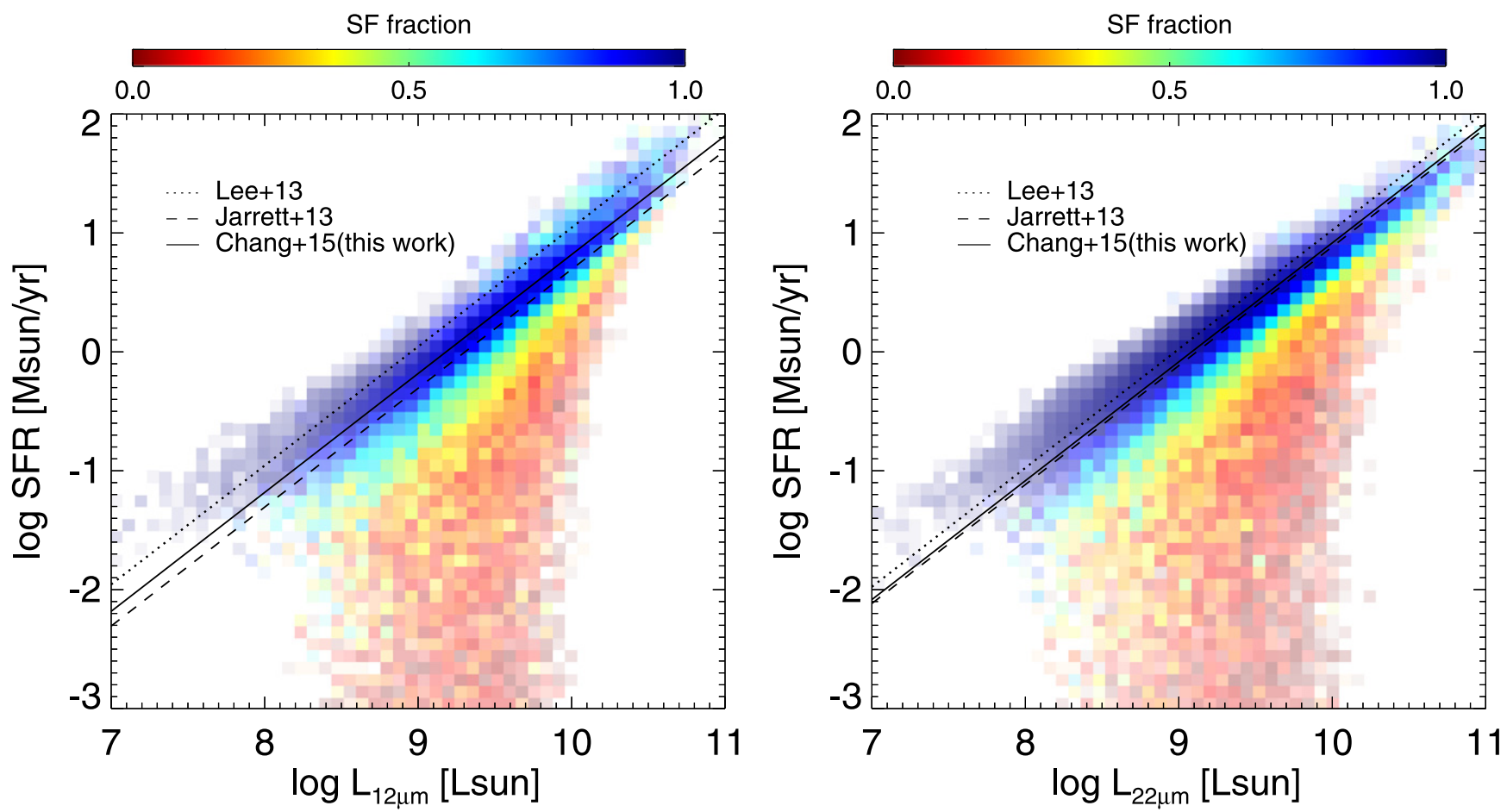

Figure 10. SFR vs. rest-frame 12 and $22 \mu \mathrm{m}$ luminosities for galaxies that have significant detections in both WISE bands. The data are binned and color-coded according to the fraction of star-forming galaxies as defined in the color-color diagram shown in Figure 2. For star-forming galaxies, we produce a linear fit as indicated by the solid lines. For comparison, we show equivalent fits from Lee et al. (2013) and Jarrett et al. (2013). The SFRs are matched to the Chabrier (2003) IMF.

completeness limit of our sample is set by the SDSS r-band spectroscopic limit for all redshifts: the fraction of galaxies above the mass limit without WISE counterparts is less than $1 \%$. The completeness limit at a given redshift is found by identifying the most massive galaxies with magnitudes near the limit. As such, we find that the mass completeness limit depends on redshift as $\log M_{\text {limit }}=10.6+2.28 \log (z / 0.1)$. The mass limit is such that actively star-forming galaxies always have significant $12 \mu \mathrm{m}$ detections; that is, if a galaxy is not detected at $12 \mu \mathrm{m}$, then it must be a quiescent galaxy. In other words, the upper limit on the SFR is useful in the sense that it allows us to distinguish between star-forming and quiescent galaxies. Using the mass limit, a galaxy of a given mass is assigned a $V_{\max }$ value assuming a survey area of 8032 square degrees. These values are given in Table 2.

In Figure 11, we show the SFR- $M_{*}$ distribution of the 363,774 galaxies above our mass limit with FLAG $=1$ (see Section 3.4). The sequence of star-forming galaxies (also known as the main sequence)-indicated in orange-is quantified by the median of 0.2 wide stellar mass bins. We fit these median values with a power law and find

$$
\log \mathrm{SFR} /\left(M_{\odot} \mathrm{yr}^{-1}\right)=0.80 \log M_{*} /\left(10^{10} M_{\odot}\right)-0.23
$$

We define the scatter in the main sequence as the $84 \%-50 \%$ range, which is 0.39 dex. The downward scatter $(50 \%-16 \%$ range) is larger $(0.64 \mathrm{dex})$, but this number is difficult to interpret due to the imperfect separation of star-forming and quiescent galaxies. This uncertainty is not (only) due to the limited fidelity in our color-color classification, but is also due to the natural variation in SF activity.

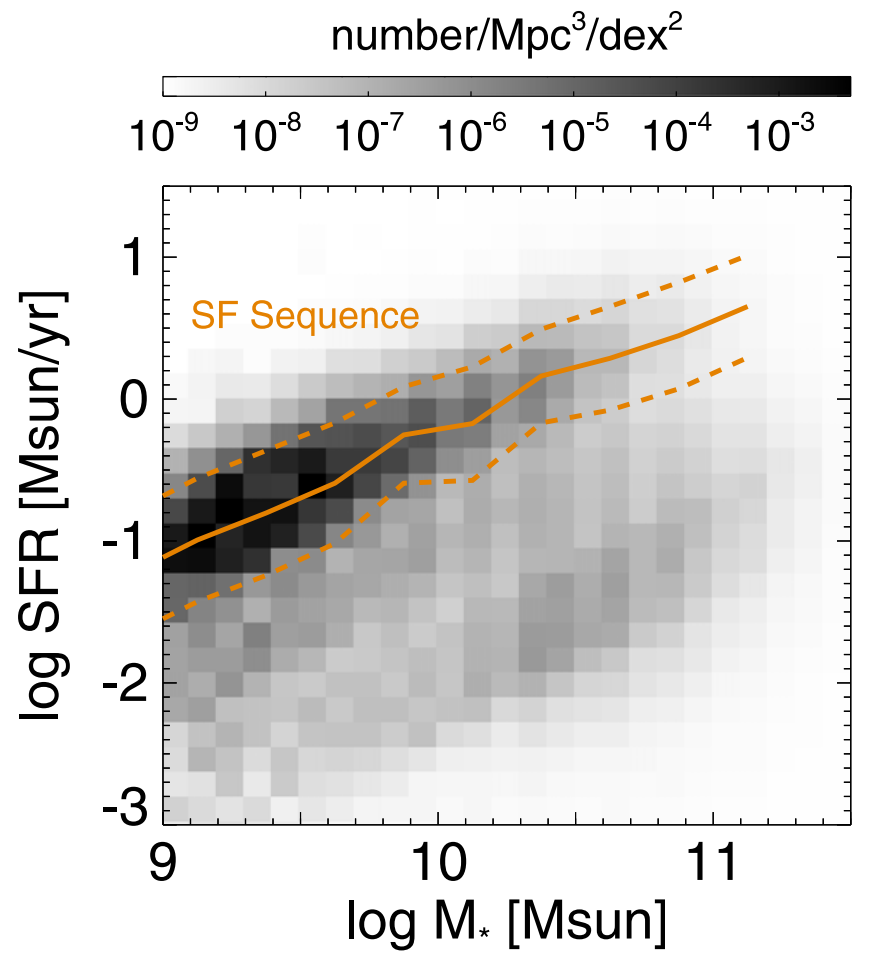

Figure 11. Distribution of galaxies above our (redshift-dependent) mass completness limit in the SFR vs. $M_{*}$ plane. The mass limit described in Section 4.3 is such that all galaxies on or near the the star-forming sequence have significant detections at $12 \mu \mathrm{m}$. The star-forming sequence is shown in orange where the dashed lines show the $1 \sigma$ scatter, as explained in Section 4.3. In order to visualize the SFR bimodality, we adopt the $1 \sigma$ upper limits on the SFR for galaxies where the SFR estimate is more uncertain than 0.39 dex. Consequently, quiescent galaxies in this plot are placed at the upper limit of the true SFR. The gray scale represents the number of galaxies after weighting by $1 / V_{\max }$. 


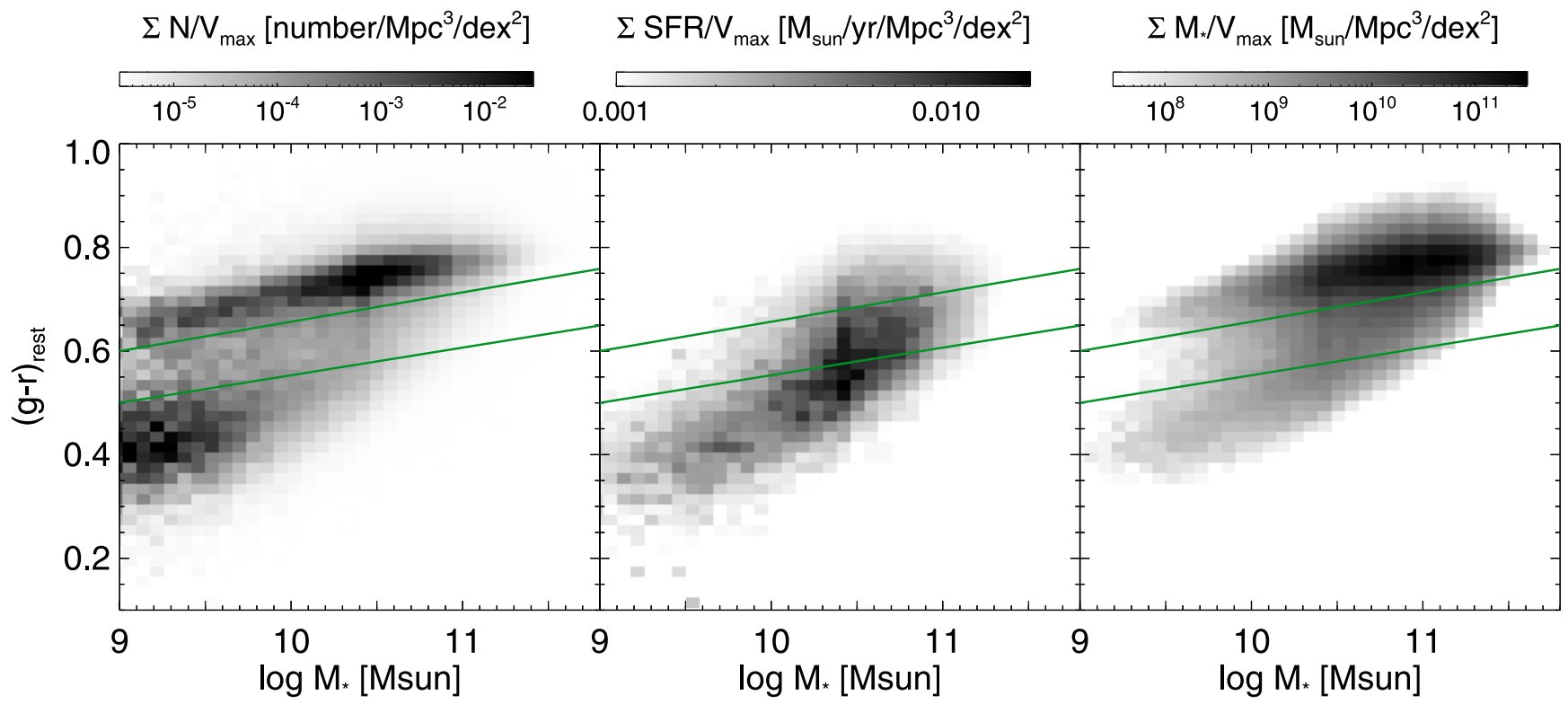

Figure 12. Left: comoving number density distribution of galaxies in the stellar mass vs. rest-frame $g-r$ plane. The gray scale reflects the numbers of galaxies in each bin after weighting with $1 / V_{\max }$. Middle: the gray scale reflects the SFR density. Right: the gray scale reflects the stellar mass density. Apparently, the green valley provides the most fertile ground for star formation as indicated by the green line.

For comparison, when we apply our fitting technique using the Brinchmann et al. (2004) values for stellar masses and SFRs, we find

$$
\log \operatorname{SFR} /\left(M_{\odot} \mathrm{yr}^{-1}\right)=0.75 \log M_{*} /\left(10^{10} M_{\odot}\right)-0.03
$$

Our fit is slightly steeper, but more importantly, has a 0.2 dex higher intercept at $M_{*}=10^{10} M_{\odot}$ and a larger scatter $(0.39 \mathrm{dex}$ versus $0.31 \mathrm{dex}$ ). The larger scatter can perhaps be attributed to the more direct measurement of highly obscured star formation when using mid-IR luminosities as a tracer as opposed to the optical emission lines. We note that the random uncertainties in our SFR estimates (typically, $0.18 \mathrm{dex}$ ) are much smaller than the scatter, indicating a large intrinsic scatter around the "mainsequence" relation. This is in stark contrast with the measurements from Brinchmann et al. (2004); their typical uncertainty is $0.30 \mathrm{dex}$ - the same as the scatter-implying zero intrinsic scatter. We conclude that the the star-forming sequence as revealed by the WISE photometry is less tight than what is inferred from optical SDSS spectra.

The bimodality seen in Figure 11 in our inferred SFR- $M_{*}$ distribution can be directly compared with Figures 17 and 24 from Brinchmann et al. (2004) where the SFRs are inferred from nebular emission lines strengths, and with Figure 15 from Salim et al. (2007) where the SFRs are inferred from UV luminosities. The color coding used in Figure 2 is based on the separation of star-forming and non-star-forming galaxies in Figure 11: non-star-forming galaxies are those that lie below the sequence by twice the scatter or more. This confirms that the two-color diagram is a simple yet very effective tool to separate dusty from passive galaxies.

Finally, it is of interest to explore the distribution of SF in relation to traditional tools to probe $\mathrm{SF}$ activity across the galaxy population, such as the color-mass diagram, the BPT diagram (Baldwin et al. 1981), and the $\mathrm{H} \delta$-D 4000 relation (Kauffmann et al. 2003). In Figure 12, we show the $g-r$ versus stellar mass distribution in three different ways: weighted by number density, weighted by SFR density, and weighted by stellar mass density. The first panel shows the well-known bimodality in the form of a red sequence and a blue cloud. We have also indicated the intermediate region often referred to as the green valley, which has been argued to contain galaxies that are transitioning from blue to red (e.g., Schawinski et al. 2007). However, as shown most recently by Taylor et al. (2015), many galaxies in this region are reddened because of dust (see also, e.g., Conselice 2006) and are not primarily due to a reduced level of SF. This is illustrated in the second panel, which shows that the galaxies in the green valley region-and not the more numerous, fainter blue galaxiesdominate the total SF budget of the present-day universe. On the other hand, these massive, star-forming galaxies do not dominate the stellar mass budget, as is shown in the third panel. The absence of a dominant population of massive, SF-ing galaxies and a general dearth of massive, disk-like galaxies (van der Wel et al. 2009) implies that their SFRs cannot be sustained for long (Schawinski et al. 2014).

In Figure 13, we show the SFR distribution in the BPT and $H \delta-D_{n}(4000)$ diagrams in three different mass bins. In the lefthand panels, we show the BPT diagram at different stellar mass bins. For the low stellar mass sample, most of the population is composed of star-forming galaxies and most SF activity also occurs in the SF region. For higher stellar mass bins, the population moves to composite galaxies and even the lowionization nuclear emission-line region, but SF activity still occurs between the star-forming and composite regions.

In the right-hand panels we see, as in Kauffmann et al. (2003), that the strength of the $4000 \AA$ break, tracing evolved stellar populations $(>1 \mathrm{Gyr})$, is anti-correlated with $H \delta$, tracing the presence of younger stellar populations (age $<1 \mathrm{Gyr}$ ). Not surprisingly, SF mostly occurs in galaxies with generally young populations. For low-mass galaxies the star-forming population is representative of the general population, but for high-mass galaxies the star-forming galaxies represent a tail of outliers, as most galaxies are old and quiescent. Still, high-mass star- 


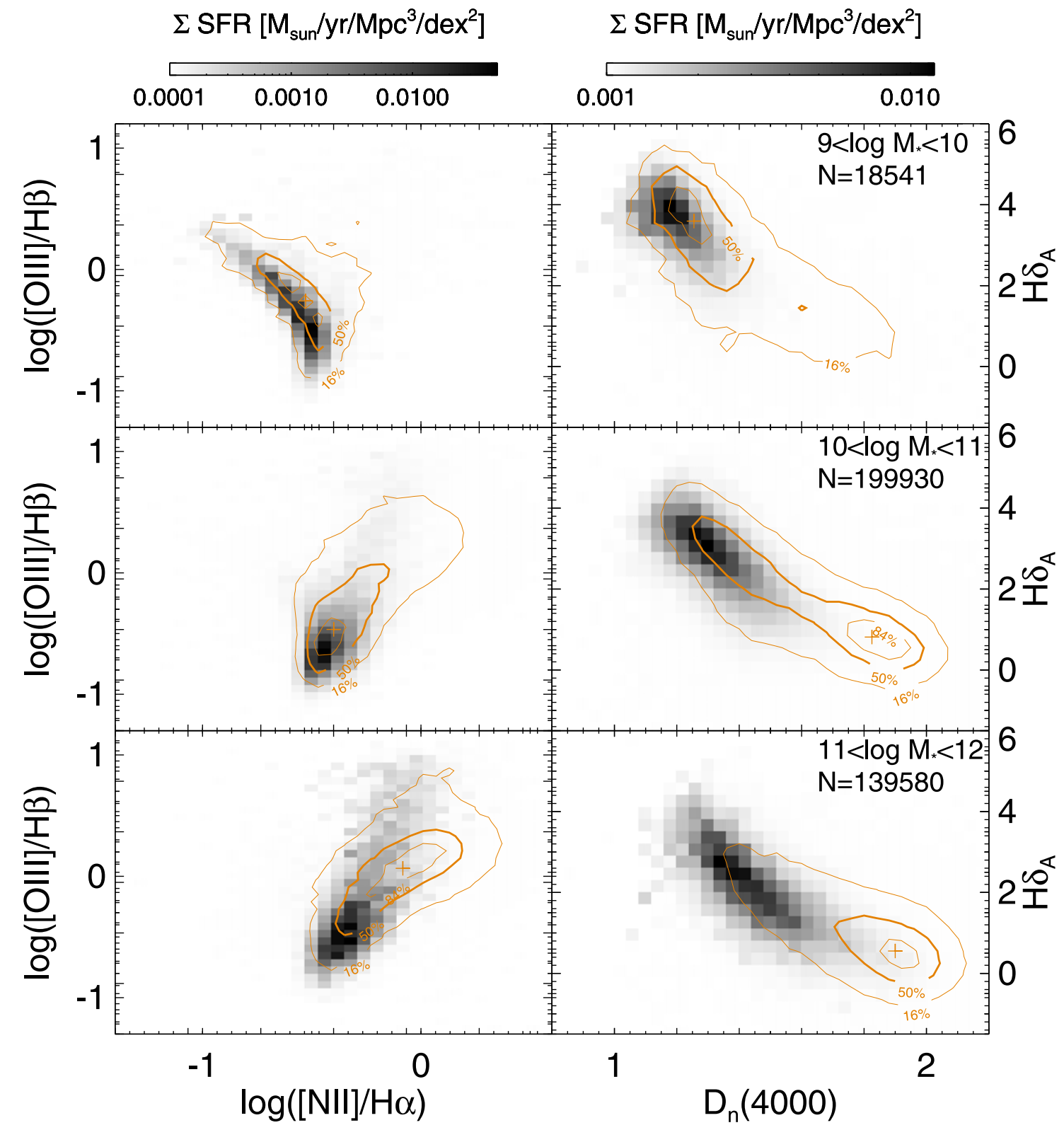

Figure 13. Number density (contours) and SFR density distribution (gray scale) in the BPT diagram (left, only galaxies with detected emission lines) and $H \delta$ $D_{n}(4000)$ (right). From top to bottom we show three different mass bins.

forming galaxies are older than their low-mass counterparts. This can be interpreted as evidence for an increasingly prominent bulge, while SF activity in the disk is similar to that in disks of lower-mass galaxies, as argued by Abramson et al. (2014). However, it could also signal a decline in SF activity as suggested by Schawinski et al. (2014).

A detailed exploration of the processes that drive SF evolution is beyond the scope of this paper, but we hope that the community will take advantage of the mid-IR-based SFR estimates published here to further distinguish between reddening by dust, age, and reduced SF activity.

\section{SUMMARY}

We revisit the measurement of stellar masses and star formation rates for the SDSS spectroscopic galaxy sample after adding four band photometry from WISE for the wavelength range $3-22 \mu \mathrm{m}$. We benefit from this wavelength extension by adopting the latest, state-of-the-art SED modeling approach (MAGPHYS; da Cunha et al. 2008), which includes the selfconsistent treatment of dust attenuation and emission and a wide range of star formation histories. The resulting SFR esimates are mostly based on PAH emission and thermal dust radiation, and therefore are complementary to the nebular emission-line-derived estimates from Brinchmann et al. (2004). In Section 4.2, we provide new calibrations for the conversion of monochromatic 12 and $22 \mu \mathrm{m}$ luminosities into SFRs.

The new $M_{*}$ and SFR estimates show the well-established bimodality in SF activity. A first application is the verification of the cruder color-color separation of star-forming and quiescent galaxies that is often invoked at higher redshifts. We confirm that the $U-V-V-J$ (or, alternatively, $u-r-$ $r-z$, as in Figure 11) color-color separation has an $89 \%$ success rate in identifying star-forming and quiescent galaxies.

We also fit the relation between SFR and $M_{*}$ (the starforming sequence, or star-forming main sequence). Our 
sequence is slightly steeper than previous studies based on SDSS galaxies, and has a higher normalization and scatter. We attribute these differences to our new SFR estimates which now include dust emission and also, to some extent, the uncertainty in establishing a purely star-forming sample (i.e., without contamination from quiescent galaxies). $L_{*}$ galaxies contribute most to the cosmic SF density, and these typically have optical colors that are between those of the traditional red squence and blue cloud (Figure 12) as a result of dust attenuation, not because of reduced star formation activity.

As a service to the community, we make publicly available our SDSS+WISE matched photometry catalog as well as the SED fitting results.

We thank the anonymous referee for useful comments which improved this paper. Y.-Y.C. was funded by the IMPRS for Astronomy \& Cosmic Physics at the University of Heidelberg and the Marie Curie Initial Training Network ELIXIR of the European Commission under contract PITN-GA-2008-214227. E.da.C. acknowledges funding through the ERC grant "Cosmic Dawn". Funding for SDSS and SDSS-II has been provided by the Alfred P. Sloan Foundation, the Participating Institutions, the National Science Foundation, the U.S. Department of Energy, the National Aeronautics and Space Administration, the Japanese Monbukagakusho, the Max Planck Society, and the Higher Education Funding Council for England. The SDSS Web Site is http://www.sdss.org/. The SDSS is managed by the Astrophysical Research Consortium for the Participating Institutions. The Participating Institutions are the American Museum of Natural History, Astrophysical Institute Potsdam, University of Basel, University of Cambridge, Case Western Reserve University, University of Chicago, Drexel University, Fermilab, the Institute for Advanced Study, the Japan Participation Group, Johns Hopkins University, the Joint Institute for Nuclear Astrophysics, the Kavli Institute for Particle Astrophysics and Cosmology, the Korean Scientist Group, the Chinese Academy of Sciences (LAMOST), Los Alamos National Laboratory, the Max-PlanckInstitute for Astronomy (MPIA), the Max-Planck-Institute for Astrophysics (MPA), New Mexico State University, Ohio State University, University of Pittsburgh, University of Portsmouth, Princeton University, the United States Naval Observatory, and the University of Washington. This publication makes use of data products from the Wide-field Infrared Survey Explorer, which is a joint project of the University of California, Los Angeles, and the Jet Propulsion Laboratory/California Institute of Technology, funded by the National Aeronautics and Space Administration. The Herschel-ATLAS is a project with Herschel, which is an ESA space observatory with science instruments provided by European-led Principal Investigator consortia and with important participation from NASA. The H-ATLAS website is http://www.h-atlas.org/.

\section{REFERENCES}

Abramson, L. E., Kelson, D. D., Dressler, A., et al. 2014, ApJL, 785, L36 Adelman-McCarthy, J. K., Agüeros, M. A., Allam, S. S., et al. 2008, ApJS, 175,297

Aniano, G., Draine, B. T., Gordon, K. D., \& Sandstrom, K. 2011, PASP, 123,1218

Baldry, I. K., Driver, S. P., Loveday, J., et al. 2012, MNRAS, 421, 621

Baldwin, J. A., Phillips, M. M., \& Terlevich, R. 1981, PASP, 93, 5

Blanton, M. R., Schlegel, D. J., Strauss, M. A., et al. 2005, AJ, 129, 2562

Bressan, A., Panuzzo, P., Buson, L., et al. 2006, ApJL, 639, L55

Brinchmann, J., Charlot, S., White, S. D. M., et al. 2004, MNRAS, 351,1151

Brown, M. J. I., Moustakas, J., Smith, J.-D. T., et al. 2014, ApJS, 212, 18

Bruzual, G., \& Charlot, S. 2003, MNRAS, 344, 1000

Chabrier, G. 2003, PASP, 115, 763

Charlot, S., \& Fall, S. M. 2000, ApJ, 539, 718

Charlot, S., \& Longhetti, M. 2001, MNRAS, 323, 887

Chary, R., \& Elbaz, D. 2001, ApJ, 556, 562

Cluver, M. E., Jarrett, T. H., Hopkins, A. M., et al. 2014, ApJ, 782, 90

Conselice, C. J. 2006, MNRAS, 373, 1389

da Cunha, E., Charlot, S., Dunne, L., Smith, D., \& Rowlands, K. 2012, in IAU Symp. 284, The Spectral Energy Distribution of Galaxies, ed. R. J. Tuffs \& C. C. Popescu (Cambridge: Cambridge Univ. Press), 292

da Cunha, E., Charlot, S., \& Elbaz, D. 2008, MNRAS, 388, 1595

Donoso, E., Yan, L., Tsai, C., et al. 2012, ApJ, 748, 80

Eales, S., Dunne, L., Clements, D., et al. 2010, PASP, 122, 499

Holden, B. P., van der Wel, A., Rix, H.-W., \& Franx, M. 2012, ApJ, 749, 96

Ibar, E., Ivison, R. J., Cava, A., et al. 2010, MNRAS, 409, 38

Jarrett, T. H., Masci, F., Tsai, C. W., et al. 2013, AJ, 145, 6

Kauffmann, G., Heckman, T. M., White, S. D. M., et al. 2003, MNRAS, 341,33

Lee, J. C., Hwang, H. S., \& Ko, J. 2013, ApJ, 774, 62

Li, H.-N., Wu, H., Cao, C., \& Zhu, Y.-N. 2007, AJ, 134, 1315

Meidt, S. E., Schinnerer, E., van de Ven, G., et al. 2014, ApJ, 788, 144

Padmanabhan, N., Schlegel, D. J., Finkbeiner, D. P., et al. 2008, ApJ, 674, 1217

Pascale, E., Auld, R., Dariush, A., et al. 2011, MNRAS, 415, 911

Peng, C. Y., Ho, L. C., Impey, C. D., \& Rix, H.-W. 2010, AJ, 139, 2097

Rieke, G. H., Alonso-Herrero, A., Weiner, B. J., et al. 2009, ApJ, 692, 556

Rigby, E. E., Maddox, S. J., Dunne, L., et al. 2011, MNRAS, 415, 2336

Salim, S., Rich, R. M., Charlot, S., et al. 2007, ApJS, 173, 267

Schawinski, K., Kaviraj, S., Khochfar, S., et al. 2007, ApJS, 173, 512

Schawinski, K., Urry, C. M., Simmons, B. D., et al. 2014, MNRAS, 440, 889

Schlafly, E. F., \& Finkbeiner, D. P. 2011, ApJ, 737, 103

Simard, L., Mendel, J. T., Patton, D. R., Ellison, S. L., \& McConnachie, A. W. 2011, ApJS, 196, 11

Smith, D. J. B., Dunne, L., Maddox, S. J., et al. 2011, MNRAS, 416, 857

Smith, D. J. B., Dunne, L., da Cunha, E., et al. 2012, MNRAS, 427, 703

Taylor, E. N., Hopkins, A. M., Baldry, I. K., et al. 2011, MNRAS, 418, 1587

Taylor, E. N., Hopkins, A. M., Baldry, I. K., et al. 2015, MNRAS, 446, 2144

van der Wel, A., Rix, H.-W., Holden, B. P., Bell, E. F., \& Robaina, A. R. 2009, ApJL, 706, L120

Vulcani, B., Bamford, S. P., Häußler, B., et al. 2014, MNRAS, 441, 1340

Wen, X.-Q., Wu, H., Zhu, Y.-N., et al. 2013, MNRAS, 433, 2946

Wen, X.-Q., Wu, H., Zhu, Y.-N., et al. 2014, MNRAS, 438, 97

Wright, E. L., Eisenhardt, P. R. M., Mainzer, A. K., et al. 2010, AJ, 140,1868

Yan, L., Donoso, E., Tsai, C.-W., et al. 2013, AJ, 145, 55

Zhu, Y.-N., Wu, H., Li, H.-N., \& Cao, C. 2010, RAA, 10, 329 